\title{
Tuberculose na grávida: um relato de caso
}

Cátia Sofia Alcobia,* Sara Alexandra Neves Costa*

\section{RESUMO}

Introdução: A tuberculose é uma doença infecto-contagiosa cuja prevalência tem vindo a diminuir em Portugal, verificando-se actualmente uma maior concentração de casos nos centros urbanos. A maioria dos casos é diagnosticada por rastreio passivo, observando-se um crescente aumento do período de tempo que medeia o início dos sintomas e o diagnóstico. Apesar de esta patologia ter uma baixa incidência nas mulheres grávidas, é importante que o diagnóstico seja atempado pelo risco de transmissão ao feto.

Descrição do caso: Mulher grávida de 32 anos que iniciou vigilância da gravidez às oito semanas de gestação na sua médica de família. Às 26 semanas é diagnosticada tuberculose pulmonar ao seu companheiro. Foi realizado o rastreio de contactos, cujo resultado foi negativo. Duas semanas depois iniciou quadro febril associado a toracalgia com evidência de derrame pleural de etiologia tuberculosa e iniciada terapêutica anti-bacilar. O parto ocorreu às 39 semanas sem intercorrências. O recém-nascido iniciou imediatamente quimioprofilaxia, tendo suspendido após confirmação de ausência de doença. A mãe manteve seguimento em consulta de pneumologia, com boa evolução clínica e laboratorial, tendo tido alta sem doença activa.

Comentário: Este relato de caso pretende enfatizar a importância do diagnóstico atempado de tuberculose na mulher grávida, cujo primeiro contacto poderá ocorrer ao nível dos cuidados de saúde primários.

Palavras-chave: Gravidez; Tuberculose; Médico de família.

\section{INTRODUÇÃO}

A tuberculose é uma doença infecto-contagiosa cuja incidência tem vindo a diminuir em Portugal; neste momento está próximo do limite definido como de baixa incidência. No entanto, a sua concentração nos centros urbanos (Porto, Lisboa e Setúbal) mantém-se com uma incidência intermédia (entre 20 a 50 casos/100.000 habitantes). Em 2013, a taxa de incidência em Portugal era de 21,1/100.000 habitantes. ${ }^{1}$

Em Portugal, $46 \%$ dos casos de tuberculose são no sexo feminino sendo os grupos etários entre os 15 e os 44 anos os mais prevalentes, coincidindo, portanto, com o período fértil da mulher. Apesar de a tuberculose ter uma baixa taxa de incidência na mulher grávida, deve existir um elevado nível de suspeição devido à sua gravidade. Os factores de risco mais comuns a ter em consideração são, por exemplo, o diagnóstico recente de tuberculose em familiares ou conviventes, a infecção pelo vírus da imunodeficiência humana, a presença de comorbilidades como diabetes, doença oncológica ou doença pulmonar obstrutiva crónica, o consumo excessivo de álcool e/ou drogas ilícitas e o facto de habitar em residências comunitárias ou sem-abrigo. ${ }^{1-2}$

A maioria dos casos de doença $(89,7 \%)$ tem sido detectada por rastreio passivo, sendo o rastreio de contactos apenas responsável por $4,5 \%$ dos casos. Aolongo dos anos tem-se observado um aumento do tempo entre o início dos sintomas

*Médicas Internas de Medicina Geral e Familiar. USF Mactamã - ACES Sintra. e o diagnóstico de tuberculose, apesar dos métodos de diagnóstico serem cada vez mais rápidos. Este facto poderá ser explicado pelo atraso entre o início dos sintomas e a procura dos cuidados de saúde por parte do utente e/ou por uma baixa suspeição por parte do profissional de saúde. ${ }^{1}$

Ao longo dos anos constata-se que uma percentagem (42\%) cada vez maior de doentes inicia tratamento apenas com base em critérios clínico-radiológicos, sendo os casos com confirmação por estudo cultural em menor número $(58 \%)$ que anteriormente. ${ }^{1}$

\section{DESCRIÇÃO DO CASO}

Grávida de 32 anos, índice obstétrico 0000, casada, empregada de balcão, Graffar III, sem antecedentes pessoais relevantes e sem história de consumos aditivos.

Iniciou vigilância da gravidez de baixo risco (segundo a escala de Goodwin modificada) às oito semanas de gestação com a sua médica de família. Nenhum dos exames realizados no âmbito de vigilância de saúde materna revelou alterações.

A gravidez decorreu sem intercorrências até às 26 semanas de gestação, altura em que recorreu à unidade de saúde em contexto de urgência por ter sido diagnosticada tuberculose pulmonar ao companheiro. No momento desta consulta já tinha iniciado seguimento no Centro de Diagnóstico Pneumológico, estando a prova de tuberculina a decorrer, que posteriormente se revelou negativa. Radiograficamente apresentava suspeita de derrame pleural 
direito que não se confirmou em avaliação ecográfica.

Duas semanas depois iniciou quadro de febre (temperatura axilar máxima $39^{\circ} \mathrm{C}$ ), tosse seca sem predomínio nocturno, mialgias e toracalgia direita. Sem cansaço ou dispneia significativos. Foi observada no serviço de urgência hospitalar, onde se verificou elevação dos parâmetros inflamatórios de fase aguda, tendo sido medicada com antibioterapia empírica. Ao $5^{\circ}$ dia de tratamento retorna ao serviço de urgência hospitalar por agravamento do quadro e início de ortopneia e trepopneia em decúbito lateral esquerdo. Na avaliação identifica-se derrame pleural à direita (1/3 inferior) (Figura 1), pelo que foi proposto internamento no serviço de infecciologia para realização de toracocentese e estudo etiológico. Perante o contexto epidemiológico e características citoquímicas do líquido pleural admitiu-se o diagnóstico de tuberculose pleural e iniciou-se terapêutica antibacilar quádrupla. Durante o internamento foi vigiada pelo departamento de obstetrícia, sem alterações do desenvolvimento fetal. Após 17 dias teve alta com melhoria clínica, laboratorial e imagiológica (Figura 2).)

Iniciou seguimento em consulta de alto risco obstétrico e manteve esquema antibacilar quádruplo até à data do parto.

$\mathrm{O}$ parto decorreu às 39 semanas sem intercorrências, apresentando o recém-nascido boa vitalidade e peso adequado (índice de Apgar 9/10/10).

$O$ recém-nascido teve seguimento em consulta de infecciologia, com início de quimioprofilaxia com isoniazida e sob aleitamento materno exclusivo. Aos quatro meses, após verificação de ausência de doença através da realização do teste de tuberculina, suspendeu antibioterapia e realizou vacinação com BCG mantendo um adequado desenvolvimento estato-ponderal e psicomotor.

A mãe manteve o seguimento em consulta de pneumologia e a toma de terapêutica anti-bacilar até completar seis meses de terapêutica, tendo tido alta com resolução.

Apesar do seguimento da gravidez ter sido realizado preferencialmente nos cuidados de saúde secundários após o diagnóstico, a mãe manteve simultaneamente o seguimento com a sua médica de família. Nestas consultas, o papel da médica foi essencialmente de suporte.

Após o nascimento, o recém-nascido cumpriu também as vigilâncias de saúde na nossa unidade de saúde, de acordo com as recomendações do Plano Nacional de Saúde Infantil e Juvenil da Direcção-Geral da Saúde.

\section{COMENTÁRIO}

A história natural da tuberculose não é alterada pela gravidez. ${ }^{3}$ Contudo, merece especial atenção nesta população pelo risco de tuberculose congénita e neonatal. Acresce o facto de se poder apresentar de forma mais in-

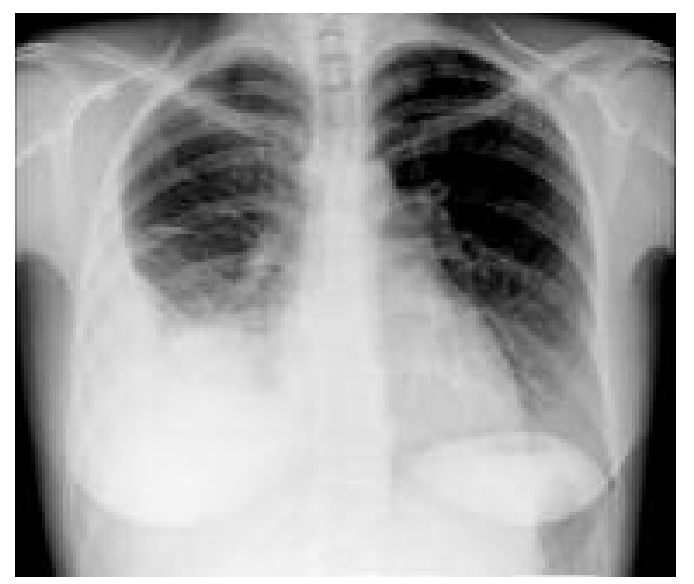

Figura 1. Tele-radiografia do tórax antero-posterior, com evidência de derrame pleural direito, à data do internamento hospitalar.

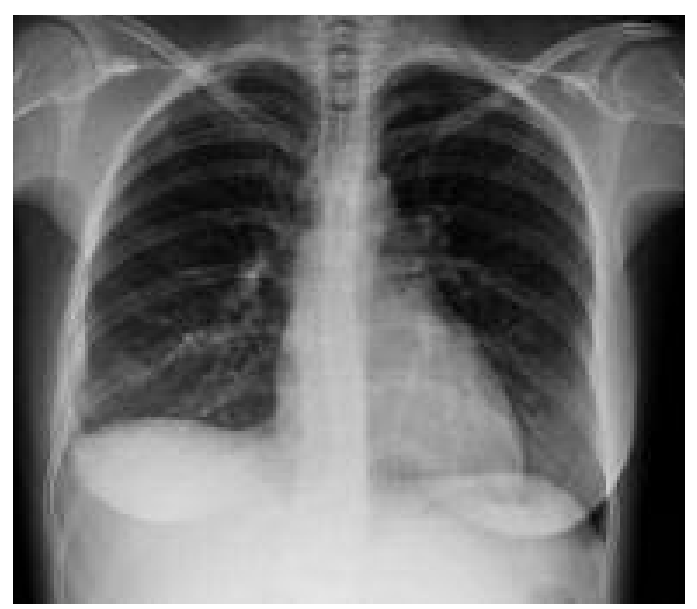

Figura 2. Tele-radiografia do tórax antero-posterior, à data da alta hospitalar.

sidiosa, uma vez que o sintoma de cansaço pode ser atribuído à gravidez e não à doença e porque a perda de peso pode ser mais difícil de reconhecer. Estas particularidades alertam-nos para a necessidade de considerar esta hipótese diagnóstica no seguimento de mulheres grávidas.

Neste caso clínico encontrava-se, como factor de risco, o diagnóstico recente de tuberculose no companheiro da nossa utente, que, por sua vez, tinha história de consumo de álcool e drogas ilícitas no passado. Após o diagnóstico do companheiro foi realizado o rastreio de contactos, cujo resultado se revelou negativo.

Neste caso observou-se um diagnóstico célere, devido ao elevado grau de suspeição existente, mesmo perante exames complementares de diagnóstico que inicialmente não confirmavam o diagnóstico. Este facto foi impor- 
tante, uma vez que o atraso no diagnóstico desta patologia, principalmente na gravidez, pode ter graves consequências quer a nível materno-fetal como social.

Perante a suspeita de tuberculose, a marcha diagnóstica deve seguir o algoritmo habitual, incluindo a tele-radiografia do tórax (assegurando apropriada protecção do feto). ${ }^{3} \mathrm{O}$ início da terapêutica não deve ser protelado, sendo que os fármacos de primeira linha - isoniazida, rifampicina e etambutol - são considerados seguros. A pirazinamida não é recomendada por alguns autores, uma vez que os seus efeitos no feto ainda não foram estudados. A estreptomicina deve ser evitada pelos seus efeitos teratogénicos. ${ }^{4}$

A amamentação deve ser encorajada nas mulheres sob terapêutica de primeira linha, porque a sua concentração no leite materno é reduzida e não apresenta toxicidade fetal. ${ }^{4}$ É fundamental ainda relembrar que a BCG está contra--indicada nos doentes com tuberculose activa, pelo que não deve ser administrada aos recém-nascidos até que se confirme a ausência de infecção. ${ }^{5}$ A necessidade de isolamento da utente não foi colocada por não estarmos perante uma situação de tuberculose pulmonar, laríngea ou brônquica. ${ }^{6}$

Ainda que a abordagem desta patologia seja da área dos cuidados de saúde secundários, o médico de família encontra-se numa posição privilegiada para suspeitar do seu diagnóstico. O conhecimento do contexto social e familiar dos utentes permite identificar mais precocemente os factores de risco para tuberculose. A possibilidade do acompanhamento continuado do utente permite ainda perceber a evolução clínica destes quadros que podem ser difíceis de suspeitar numa primeira avaliação.

\section{REFERÊNCIAS BIBLIOGRÁFICAS}

1. Programa Nacional para a Infeção VIH, SIDA e Tuberculose. Portugal: infeção VIH, SIDA e tuberculose em números [Internet]. Lisboa: Direcção-Geral da Saúde; 2014. Available from: https://www.dgs.pt/estatisticas-de-saude/estatisticas-de-saude/publicacoes/portugal-infecao-vih-sida-e-tuberculose-em-numeros-2014.aspx

2. Secção de Neonatologia da Sociedade Portuguesa de Pediatria. Protocolos de diagnóstico e terapêutica em infecciologia perinatal [Internet]. Porto:Angelini Farmacêutica; 2007. Available from: http://www.spp.pt/UserFiles/ File/Infecciologia_Perinatal_2007/Infecciologia_Perinatal.pdf

3. Friedman LT, Tanoue LN. Tuberculosis in pregnancy [Internet]. UpToDate; 2016 [updated 2016 Aug 23]. Available from: http://www.uptodate.com /contents/tuberculosis-in-pregnancy?source=search_result\&search=tuberculosis+in+pregnancy \&selectedTitle $=1 \% 7 \mathrm{E} 14$

4. Centers for Disease Control and Prevention. Tuberculosis and pregnancy. CDC; 2016 [cited 2015 Dec; updated 2014 Dec 18]. Available from: http://www.cdc.gov/tb/topic/populations/pregnancy/default.htm

5. Direcção-Geral da Saúde. Plano nacional de vacinação: norma de orientação clínica nº 040/2011, de 21/12/2011. Lisboa: DGS; 2012.

6. Instituto Nacional de Saúde Dr. Ricardo Jorge. Recomendações para as precauções de isolamento precauções básicas e dependentes das vias de transmissão [Internet]. Lisboa: INSA; 2007 [cited 2012 Oct]. Availa-ble from: http://www.dgs.pt/paginaRegisto.aspx?back=1\&id=12427

\section{CONFLITOS DE INTERESSE}

As autoras declaram não ter conflitos de interesse.

\author{
ENDEREÇO PARA CORRESPONDÊNCIA \\ Cátia Sofia Alcobia \\ USF Mactamã - ACES Sintra \\ Rua Teixeira Pascoais \\ 2745-852 Massamá \\ E-mail: catia.alcobia@gmail.com
}

Recebido em 15-02-2016

Aceite para publicação em 22-09-2016

Artigo redigido ao abrigo do acordo ortográfico anterior a 1990.

\section{ABSTRACT}

\section{TUBERCULOSIS IN PREGNANCY: A CASE REPORT}

Introduction: Tuberculosis is an infectious disease with a declining prevalence in Portugal, with more cases reported in urban centres. Most cases are diagnosed by passive screening but there are reports of increasing delay between the onset of the symptoms and diagnosis. Although this disease is rare in pregnant women, early diagnosis is important, because of the risk of vertical transmission to the fetus.

Case Description: We present the case of a 32 year-old woman who began follow-up of her pregnancy with her family doctor at eight weeks gestation. Her partner was diagnosed with pulmonary tuberculosis during the 26th week of gestation. She was referred for contact screening which was negative. Two weeks later she presented with fever and thoracic pain with a tubercular pleural effusion. She started tuberculostatic therapy. Delivery was at 39 weeks without complications. The infant received immediate chemoprophylaxis that was stopped after confirming the absence of disease. The mother was followed by a pneumologist with good clinical and laboratory resolution, and was discharged from care without active disease.

Comments: This case report demonstrates the importance of early diagnosis of tuberculosis in pregnancy. The first contact with the patient may occur in primary care.

Keywords: Pregnancy; Tuberculosis; Family physician. 